\title{
ÁDÁM SZINGER
}

\section{AN DER GRENZE VON LITERATUR UND WIRKLICHKEIT: ÜBERLEGUNGEN ZU THOMAS BERNHARDS "MEINE PREISE"}

\begin{abstract}
Ich nahm meinen Zettel mit meinem Text aus der Rocktasche und verlas ihn, möglicherweise mit zittriger Stimme, kann sein. Auch die Beine bebten mir, naturgemäß. Aber ich war noch nicht zuende mit meinem Text, als der Saal unruhig wurde, ich wußte gar nicht warum, denn mein Text war von mir ruhig gesprochen und das Thema war ein philosophisches, wenn auch von einiger Tiefgründigkeit, wie ich fühlte und ein paarmal hatte ich das Wort Staat ausgesprochen. (Bernhard 2009: 81 f.)
\end{abstract}

Thomas Bernhards posthum veröffentlichter Band "Meine Preise“ ist im Jahre 2009 zum 20. Todestag des österreichischen Schriftstellers als eine Überraschung erschienen. Nähere Angaben und Erläuterungen zur Herausgabe kann man in der editorischen Notiz, einem Nachwort zum Werk, von Raimund Fellinger, dem Präsidenten der Internationalen Thomas Bernhard Gesellschaft erhalten. Aus dem Nachlass wissen wir, dass das Typoskript "Meine Preise" schon Anfang der 1980er Jahre entstand, (vgl. Bernhard 2009: 132) und es gibt Hinweise darauf, dass Bernhard die Texte selbst zur Ausgabe bestimmte: Bei einer Begegnung am 23. August 1988 in Ohlsdorf berichtete er seinem Verleger Siegfried Unseld über ein Prosawerk, das er laut Vereinbarung im März 1989 hätte abgeben sollen, was aber wegen seines Todes nicht verwirklicht werden konnte (vgl. Bernhard 2009: 131). Es gibt Hypothesen in Bezug darauf, warum Bernhard den Band zu Lebzeiten nicht hat publizieren lassen, "obwohl er dazu jede Verlagsmöglichkeit gehabt hätte“ (Huber 2016: 14). Vermutet wird, dass er das Manuskript im Bewusstsein seiner Krankheit für spätere Zeiten zurückbehalten hat (Sarankó 2009), oder dass es in einem ,spekulativen' Zusammenhang „mit dem Nobelpreis für den ,Rivalen' Elias Canetti (1981) steht (vgl. Huber 2016: 34), diese Vermutungen sind aber nicht weiter belegt. Zur Werkgeschichte gehört ferner, dass der Publikation ein Vorabdruck der Kleingeschichten in der Frankfurter Allgemeine Zeitung vorausging, der eine erste Rezeptionswelle in Deutschland und in Österreich auslöste. Die Missverständnisse im Zusammenhang mit Bernhard lassen sich in Deutschland aus den Reaktionen der Rezipienten ablesen. Der erste Widerspruch kam von Arnold Stadler: Er bemerkt, dass in der Geschichte um die Regensburger Staatsverleihung nur die Hälfte stimmt. Dann verfällt Maxim Biller einem „populären Irrtum“ (Huber 2016: 13), indem er sagt, dass Bernhard „in keinem seiner Bücher so ehrlich“ war. Eine positive Kritik kam vor allem erst aus Österreich (vgl. Huber 2016: 12 ff.). Dass die Herausgabe des Buches sowie dessen Publikumserfolg das Interesse für 
Bernhard reaktivierte, belegen Buchpräsentationen, Symposien und Tagungen der kommenden Jahre. Die Wirkung war ein Ergebnis der bewussten Strategie: Die Übersetzungen kamen dank der österreichischen finanziellen Hilfe und der kulturellen Mitarbeit gleichzeitig mit dem Original heraus. (Bombitz 2019: 38 ff.; Pecka 2010: 139). Außer Frage steht auch der Erfolg, wenn man die Zahlen des Literaturmarkts betrachtet: „Mit Stand Mai 2016 wurden über 55000 Exemplare verkauft, Übersetzungen in über 20 Sprachen lizensiert" (Huber 2016: 33).

\section{Thomas Bernhard und Österreich}

In der frühen Rezeption des Österreichers war das literarische Werk von den öffentlichen Auseinandersetzungen nicht zu trennen. Bernhard war als politischer Autor angesehen, der in der allgemeinen apolitischen Grundhaltung der 1960er und 1970er Jahre in Österreich seine Kritik in einem beinahe leeren Raum widerschallen ließ. Wie prägend diese ,Einstufung' für die kommenden Zeiten war, lässt sich am Beispiel der frühen Aufnahme Bernhards in Ungarn und in Tschechien ablesen: Sowohl für die tschechischen als auch für die ungarischen Autorenkollegen war er anfangs der politische Autor, ein Organ oder eine Stimme, mit der man die aktuelle politische Regierung kritisieren konnte. Die zu der Zeit herausgebildete Selbstnarrative dient, wie es später ausführlicher diskutiert wird, der rhetorischen Basis des Werkes "Meine Preise“.

Dass Bernhard ein streitbarer Autor wurde, war größtenteils von den kulturellen Zuständen der 1950er und 1960er Jahre, beziehungsweise von kulturpolitischen Maßnahmen der österreichischen Regierung der Zweiten Republik abzuleiten. Im 20. Jahrhundert boten sich zwei Identifikationsebenen für Österreich, die mit den Brüchen der Geschichte wegfielen: Die Monarchie zerfiel nach 1918, die Zugehörigkeit zum Deutschtum wurde nach 1945 unvorstellbar. Das Land gelangt innerhalb von 5 Jahrzehnten von einem Reichsbewusstsein - Austria erit in orbe ultima - bis zum finis Austriae, dem Anschluss (vgl. Reuter 2013: 53) Die österreichische Identität war in den Zeiten nach dem Zerfall des Reiches selbst ein Anachronismus, denn das Einzige, das diese Brüche überleben konnte, war der habsburgische Mythos ${ }^{2}$, der durch Idealisierung von Joseph Roth, Franz Werfel, Stefan Zweig, Robert Musil oder Heimito von Doderer nach dem Untergang der Monarchie "in seine eindrucksvollste und interessanteste Phase" (Reuter 2013: 77) trat.

Bei Reuter erfährt man ferner, dass nach dem Ersten Weltkrieg auch ein verstärktes deutsches Nationalgefühl vorherrschend wurde: Es habe z. B. einen Anspruch auf einen Anschluss zu Deutschland gegeben, der unter dem Großteil der Bevölkerung Unterstützung fand (Österreich hätte sich Deutschösterreich nennen lassen), welchen Wunsch aber die Sieger verboten. Es gibt eine inte-

1 Zum Thema mit ungarischer Relevanz siehe ferner: Bombitz (2006); Pecka (2010).

2 Zum Thema siehe ausführlich: Magris (2000). 
ressante und vielsagende Angabe zu diesem Thema auch aus den 1950er Jahren: Angeblich haben sich „46 Prozent der Bevölkerung noch 1956 bei einer Meinungsumfrage [...] für , die Zugehörigkeit zum deutschen Volk' entschieden“ (Reuter 2013: 65).

Das Identitätsproblem kulminiert aber erst richtig mit den kulturpolitischen Konsequenzen des Staatsvertrags und des Neutralitätsgesetzes von 1955. Österreich wurde zum Opfer des Dritten Reiches deklariert und ein staatlich geförderter Prozess der Nationalbildung wurde auf deren Basis in Gang gesetzt. Die Kulturpolitik der Identitätsbildung rief eine weitere, noch tiefere Krise hervor. Mit den Maßnahmen wurden nämlich die von den Nazis verfolgten Austro- und Klerikalfaschisten nach der Nazi-Zeit auch als Opfer betrachtet, die zu jenen Positionen gelangten, die sie bis 1938 innegehabt hatten. „All das, was von den Nazis vertreten und durchgesetzt wurde, wurde von innen patriotisch umformuliert aber nicht zurückgenommen“ (Reuter: 2013: 79). Die Ideen des Faschismus setzten sich verborgen in der Zweiten Republik fort. Von nun an existierten „eine öffentlich vertretene Opferschaft und eine verheimlichte Täterschaft" (Reuter 2013: 79). ${ }^{3}$ Das im 20. Jahrhundert mehrfach belastete Österreich-Bild wurde mit dieser einseitigen (falschen) Narrative weiter geschwächt und verlor viel an Identifikationspotential.

Bernhard war ein Künstler, der mit der apolitischen Haltung und mit dem falschen Identifikationsbild abrechnet. Schon in seinem frühen Werk in den 1950er Jahren lassen sich eine Abwendung von der idyllischen Heimatliteratur - d. h. dem großväterlichen Erbe - und eine Hinwendung zum Genre der AntiHeimatliteratur erkennen. Für diese Tendenz ist seine Erzählung mit dem Titel „Der Schweinehüter" aus der Mitte der 1950er Jahre emblematisch, die 1956 veröffentlicht wurde, und die im Keime schon seine neue Stimme andeutete. In seinem Frühwerk „Frost" (publiziert 1963), das das literarische Feld für die späteren politischen Affären vorbereitet, wird bereits ein Spannungsfeld zwischen der schönen Landschaft Österreichs und dessen unfähiger, inkompetenter Staatsverwaltung thematisiert. In diesem Bezug ist ferner ein Autor namens Hans Lebert zu erwähnen, der mit seinem Werk "Wolfshaut" (1960) als Vorbild für die Anti-Heimatliteratur dient.

Den Presseartikel „Politische Morgenandacht” (1966) als eine Ausnahme genommen und daher außer Acht gelassen, kann man behaupten, dass sich die Epoche der politischen Auseinandersetzungen zwischen Bernhard und der Regierung sowie dessen Vertretern zwischen 1968 und 1979 datieren

3 Es ist kein Wunder, dass eines der Bernhard'schen ,Leitmotive' die Kritik der NS-Ideologie wurde. Das Thema kehrt in "Meine Preise“ auch an verschiedenen Stellen zurück: Als Grund zum Austritt aus der Akademie für Sprache und Dichtung, sowie als organischer Teil der Geschichte „Der Literaturpreis der Freien und Hansestadt Bremen“. Zum Thema siehe ferner: Judex (2016). 
lässt. ${ }^{4}$ (Mittermayer 2006: 52) Dafür gibt es zwei Gründe: Einerseits lässt sich behaupten, dass der erwähnte Artikel über eine starke Metasprache verfügt, die noch rein, d. h. mit keiner Objektsprache vermischt erscheint, die so (mehr philosophisch als konkret ausgestattet) wenig Reaktion hervorrufen konnte. Andererseits ist das Anfangsdatum der Periode sowohl im politischen, als auch im gesellschaftlichen Sinn symbolisch. Die Revolte von 1968 gestaltete sich in Österreich mehr in der Kunst und nicht auf der Straße (vgl. Mittermayer 2006: 52). Es war eine soziale Gegebenheit, wodurch Bernhards politische Äußerungen ein weiteres Lesepublikum und eine gewisse Popularität gewannen. Es ist keine Überraschung, wenn man bedenkt, dass die Revolte gar keine Bewegung war, denn sie verfügte über keine bürgerliche Basis: Die Arbeiter konnten sich mit den Ideen der Studenten nicht identifizieren (vgl. Schmidt-Dengler 1995: 216 f.). Für das Werk „Frost" erhielt Bernhard am 22. März 1968 den Österreichischen Staatspreis. Er ,berichtet' über diese Ereignisse ausführlich in "Meine Preise“, wo auch seine Ansprache als Begleittext zu finden ist. Darin steht $u$. a. bereits Folgendes: Die Österreicher sind "apathisch" und "Geschöpfe der Agonie“, "das Zeitalter ist schwachsinnig, der Staat ist ein Gebilde, das fortwährend zum Scheitern, das Volk ein solches, das ununterbrochen zur Infamie und zur Geistesschwäche verurteilt ist" (vgl. Bernhard 2009: 121 f.). Um an der Stelle einen weiteren Kontext zu gewinnen, bietet sich der Vergleich dieser Zitate mit dem Motto dieser Arbeit an, denn sie haben denselben Bezugspunkt im referenziellen Bereich.

In den 1970er Jahren, und besonders in der zweiten Hälfte des Jahrzehntes vermehren sich Bernhards Auseinandersetzungen mit Personen und Angriffe auf diese, „über deren Ansehen womöglich allgemeiner Konsens besteht" (Mittermayer 2006: 63).

In einem Artikel in der "Zeit" schreibt Bernhard mit dem Titel „Was Österreich nicht lesen soll“ Folgendes:

Die Regierungen, die wir in den letzten Jahrzehnten gehabt haben, waren zu jedem Verbrechen an diesem Österreich bereit, und sie haben an diesem Österreich jedes nur denkbare Verbrechen begangen [...].

und

Das Parlament des heutigen Österreich ist, auf dem politischen Unrat in diesem Lande, ein luxuriöser und kostspieliger, lebensgefährlicher Wurstelprater, und die Regierung ist eine ebenso teure Dummköpfelotterie. Wenn der Vorhang des Staates aufgeht, sehen wir an jedem österreichischen Tag (und als auch am Nationalfeiertag) ein Lustspiel für Marionetten. Wenn wir genauer hinschauen,

4 Man soll sich vor Augen halten, dass Bernhard für seine Tätigkeit einen Raum schafft, wo er eine ,Literarisierung' der Wirklichkeit bzw. eine ,Realisierung' der Literatur treibt. Das heißt einerseits, dass Bernhard keine ,Geschichte' schreibt, und andererseits, dass man seine politischen Äußerungen immer in einem weiteren literarischen Kontext lesen soll. 
sehen wir, was wir immer gesehen haben: Die Marionetten sind das schwachsinnig unbelehrbare Volk, und die daran ziehen (die Drahtzieher), die das Volk für dumm verkaufende Regierung. (Bernhard 1978)

Die Unruhe, die Bernhard mit seinen Scheltreden und Hasstiraden in der Öffentlichkeit und mit seinen Schriften stiftete, war auch in Österreich abzulesen. Es war z. B. fraglich, ob man das neue Genre der Anti-Heimatliteratur als eine nationale Literatur annehmen kann, und ob es, wenn es die neue österreichische Literatur ist, überhaupt ein gewisses Identifikationspotenzial hat. Robert Menasse fügt dieser Frage die Bemerkung hinzu:

In Österreich [...] wird die österreichische Literatur gemeinhin auf dieselbe Weise gelesen und nach den selben allgemeinen ästhetischen Kriterien überprüft, wie jene, die aus Irland, Deutschland, Frankreich oder den USA kommt - das heißt, in Österreich wird die österreichische Literatur ohne Umwege gleich als Weltliteratur konsumiert. (Menasse 2005: 117)

Am ersten Todestag von Bernhard schreibt der deutsche Literaturkritiker Marcel Reich-Ranicki die folgenden Zeilen, die mit den Beobachtungen von Menasse gewissermaßen in Einklang gebracht werden können:

Wie, wenn er, der doch in einem holländischen Ort geboren wurde, dort aufgewachsen wäre? Kann man sich ihn als einen holländischen Schriftsteller denken? Gewiß, nur wären dann für ihn, ich bin dessen sicher, die Niederlande eine einzige „geist- und kulturlose Kloake“, dann hätten die Holländer von ihm allerlei Böses über, sagen wir, Rembrandt hören müssen. Kurz und gut: einem holländischen Autor Thomas Bernhard hätte natürlich nicht Österreich als Metapher für Sinnlosigkeit unseres Daseins gedient, sondern Holland. (zitiert nach Reuter 2013: 54)

Diese Äußerung löste eine heftige Debatte aus, Irmtraud Götz von Olenhusen schreibt in ihrer Replik Folgendes:

Die Verstrickung Österreichs in die nationalsozialistischen Gewaltverbrechen sowie die Unfähigkeit der österreichischen und deutschen Gesellschaft, damit umzugehen, war für Thomas Bernhard zentrales Moment seiner nationalen Identität als deutschsprachiger, österreichischer Schriftsteller. Er war nicht ein in Österreich lebender Holländer, sondern ein in Holland geborener Österreicher, der mit seiner Biographie, seinen negativen Identifikationen mit diesem Land, mit der Realität von Vergangenheit und Gegenwart und nicht nur metaphorisch mit der Sinnlosigkeit des Daseins zu tun hatte; die Sicht des Autors war durch Zeitumstände, Orte und Geschehnisse bedingt, und seine negative Liebe zu Österreich hat, wenn man so will, mit Identitätssuche oder mit expliziter Identitätsverweigerung zu tun. (zitiert nach Reuter 2013: 55) 
Die historischen Ereignisse und die dazu gehörenden Debatten und Diskussionen, wie kurz dargestellt, dienen als historisch-kultureller Hintergrund zu "Meine Preise" und stehen zu der heiteren Stimmung des Werkes als ernstliches Komplement, das zu den kurzen Geschichten über die behandelten Preisverleihungen auch mit einem dunklen Kolorit beiträgt. Die Referenzialität auf die Wirklichkeit wird durch vier kurze Begleittexte in "Meine Preise" gestärkt: Die „Ansprache bei der Verleihung des Literaturpreises der Freien und Hansestadt Bremen", die "Ansprache bei der Verleihung des Österreichischen Staatspreises", die "Ansprache bei der Verleihung des GeorgBüchner-Preises" sowie ein kurzer Text mit dem Titel "Zu meinem Austritt". Betrachtet man die ,Funktion' und die Sprache dieser Texte, kann man feststellen, dass sie in diesem Hinblick auf die Kommunikation einen engeren Bezug auf die ,reale Welt' haben, schon von der Tatsache her, dass sie Zeitgenossen direkt ansprechen, also über eine reale persönliche Intonation verfügen. Es besteht eine Kluft zwischen dem Stil dieser Texte und dem der ,Haupttexte' in "Meine Preise": Was die Sprache dieser Texte betrifft, ist feststellbar, dass sie eine gewisse Ähnlichkeit mit der Sprache der erwähnten Zeitungsartikel („Politische Morgenandacht", "Was Österreich nicht lesen soll“) aufweist. Denn es geht hier wiederum um eine starke Metasprache, die nichts Näheres und gar nichts Konkretes ,besagt', aber existenzielle, philosophische Fragen anspricht. Man kann die Preisverleihungsgeschichten, die den Großteil von „Meine Preise“ ausmachen, als persönlichen Kommentar zu den Ansprachen sowie zu den kulturell-politischen Gegebenheiten der Nachkriegszeit in Österreich lesen, und sich dabei - heute aus einer zeitlichen Entfernung - sogar amüsieren.

\section{„Meine Preise“}

Laut Raimund Fellinger lassen sich die kurzen Geschichten in Meine Preise als die Fortsetzung der autobiographischen Pentalogie und auch als Vorläufer der Erzählung „Wittgensteins Neffe" lesen, die eine Zeitspanne zwischen 1963 und 1980 umfassen und zur persönlichen Chronik des Lebens Bernhards werden. Das Konvolut im Nachlass besteht aus verschiedenen Texten, die Bernhard selbst zur Publikation vorbereitet hatte: Kleine ,Storys' über Preisübergaben und Ansprachen zu den Festlichkeiten.

In neun mehr oder weniger kurzen Geschichten erzählt Bernhard über die Umstände und Verhältnisse seiner Preisübernahmen in einem Zeitraum von 1964 bis 1976, wo verschiedene ,Bewegungen' der Texte abzulesen sind. Merkwürdig ist z. B., dass die ,Berichte' mit der Entgegennahme des Grillparzerpreises (1972) beginnen, die im temporalen Mittelpunkt der erzählten Zeitperiode steht. Die folgenden drei Texte reichen bis 1964 zurück, und diese Erzählweise macht die Erzählstrategie dieser Texte der der autobiographischen Pentalogie, wo die Lebensereignisse auch rückgängig erzählt 
werden, ähnlich ${ }^{5}$. Als Höhepunkt steht „Der Österreichische Staatspreis für Literatur" - die meist zitierte und skandalöseste Preisverleihungsgeschichte -, ausgerechnet in der Mitte der Storys. Wenn man bedenkt, dass „Der Österreichische Staatspreis für Literatur" mit zwanzig Seiten auch von der Länge her die bedeutendste Geschichte ist, und dass Bernhard in dieser am stärksten seine Selbstnarrative schreibt, kann man den symbolischen Wert der zentralen Position dieser Geschichte auch nicht übersehen. Bleibt man nun bei dieser Symbolik und betrachtet die Position (die letzte) und Seitenanzahl (kaum mehr als fünf Seiten) der Geschichte des Büchnerpreises, der der international renommierteste Preis ist, wird einem auffallen, dass Bernhard seiner Verachtung ${ }^{6}$ auch Form stiftet.

Dann geht die Richtung der erzählten Zeit vorwärts bis 1976, um schließlich, wie bei der Bewegung eines Pendels, in der Nähe des temporalen Mittelpunkts (1972) zu verharren.

Motivisch bewegen sich die Kurzgeschichten um die Beziehung des Erzählers zum Preis herum: Es besteht ein ambivalentes Verhältnis des Geld-HabenWollens und des Verachtens des Preises. So wird „dem Preis als literarischem, politischem und wirtschaftlichem Diskurs [...] je eine phänomenologische, existentialistische und morphologische Strategie zuerkannt“ (Bombitz 2019: 38 f.). Diese Leitmotive werden dann um nebensächliche Begebenheiten, die zu Anekdoten erhoben wurden, ergänzt und erweitert. So erfährt man, was den Erzähler motiviert, die Unannehmlichkeiten, die bei einer Preisverleihung entstehen, zu überstehen: ein beinahe unbewohnbares Haus mit großen Mauern oder ein neues Sportauto, woran nach drei Monaten bei einem Verkehrsunfall im Ausland ein Totalschaden entsteht. Eins bleibt aber thematisch ständig präsent, und das ist die Geldgier:

So war ich, zugegeben, immer bei dem Gedanken an die Preissumme von fünfundzwanzigtausend mit dem Preis einverstanden, mit allem Scheußlichen und Widerwärtigen, das mit dem Preis im Zusammenhang stehen musste, ich verabscheute den Preis immer nur so lange ich nicht an die fünfundzwanzigtausend Schilling dachte, dachte ich an die fünfundzwanzigtausend Schilling, fügte ich mich in mein Schicksal. (Bernhard 2009: 69 f.)

5 „Bernhards Strategie der,Geschichtenzerstörung`vermeidet narrative Folgerichtigkeit [...], um die biographischen Bruchstücke konstellativ anzuordnen." (Klug 1991: 255)

6 „In seinen späteren Lebensjahren nimmt Bernhard keine Preise mehr an; unter den Auszeichnungen, die ihm verliehen worden wären, sind so renommierte wie der Premio Prato (1982), Premio Mondello (1983) und der Premio Antonio Feltrinelli (1987) sowie der Prix Médicis (1988)“ (Mittermayer 2006: 57). 
Auffallend ist der Stil dieser Geschichten im Hinblick auf die Tatsache, dass Bernhard sich gern als der große Geschichtenzerstörer postuliert, denn hier geht es wohl um Anekdoten und heitere Nostalgie, wo die von Bernhard in anderen Texten sonst so gern verabscheuten Redewendungen auch nicht erspart bleiben:

Das war das erste Interview meines Lebens, möglicherweise gab ich es dem Hamburger Abendblatt, wer weiß. Ich war so aufgeregt, daß ich keinen einzigen Satzvollständig zuende sagen hatte können, auf alle Fragen wußte ich zwar sofort eine Antwort, aber ich war nicht glücklich über meine Formulierungskunst. Ich dachte, die Leute merken jetzt, daß du aus Österreich kommst, wo die Füchse Gutenacht sagen. ${ }^{7}$ (Bernhard 2009: 53 f.)

Obwohl eine starke ,Lebensbezogenheit' nachweisbar ist und einige Belege besonders im Fall von "Der Österreichische Staatspreis für Literatur" dafür stehen $^{8}$, darf man nicht leichtsinnig in die Falle gehen, indem man sich denkt, dass Bernhard jetzt einen ehrlichen Einblick in sein Privatleben erlaubt, denn in "Meine Preise" hat man ebenso wie im Fall der autobiographischen Schriften mit einer starken Stilisierung und mit einem doppelt kodierten Bild zu tun: Fiktion und Wirklichkeit, Realität und Literatur vermischen sich miteinander in den Geschichten, und die Wirkung ist mit einer kalkulierten Rhetorik vorbereitet. Die Publikumserwartungen sind natürlich durch dieses ,Missverständnis wohl bedient: Der Titel selbst und die Tatsache, dass "Meine Preise“ aus dem Nachlass publiziert wurde, bereiten den Weg zu einer objektiven Lektüre vor. Wie stark die ,Verführungskraft' dieses Stils ist, zeigt, dass es viele Kritiken gibt, wo die RezensentInnen die Texte für bare Münze nehmen (vgl. Huber 2016: $12 \mathrm{ff}$.$) .$

Sprachlich-stilistisch knüpfen also diese kleinen Geschichten an die autobiographischen Schriften enger an, die sarkastischen Anekdoten bewegen sich im Spannungsfeld desselben Referenzbereichs. Um die Stilisierung hinter den Geschichten von "Meine Preise“" zu entlarven, bietet sich die Möglichkeit, gleiche Textstellen in diesem Werk und in "Wittgensteins Neffe" zu untersuchen: Die durch ein Zitationsspiel verdoppelten Geschichten sind „Der Grillparzerpreis" beziehungsweise "Der Österreichische Staatspreis für Literatur", deren Ereignisse in "Wittgensteins Neffe" eng nacheinander beschrieben sind. (s. Bernhard 2015: 105 ff.) Die kleineren Unterschiede, die auf die leichte Stilisierung stark verweisen, sind amüsierend: Ist der Herr Paul Wittgenstein wirklich Begleiter des Erzählers? Kauft der Erzähler im Warengeschäft Sir Anthony den neuen Anzug alleine oder in Gesellschaft? Ist die vom Minister Piffl-Perčević zugeschlagene Glastür in tausende Scherben zersplittert oder nicht? Wenn

7 Die letzte Hervorhebung von Á. Sz.

8 Zu einem Vergleich siehe Bernhard/Unseld (2009: 65 ff.); Hennetmair (2000: 29 f., 46, $59,62)$. 
man die Geschichten einer näheren Untersuchung unterzieht, kommen solche Widersprüche ans Tageslicht. Die Frage ist aber, ob man diese Abweichungen in der Darstellung wirklich für Widersprüche halten muss? Sind sie nicht eher Variationen derselben Geschichten, die auf eine Literarisierung der Wirklichkeit hinweisen? Eine mögliche Interpretation dieses Phänomens ist, dass Bernhard "Meine Preise" durch dieses Zitationsspiel in die Reihe der autobiographischen Schriften stellt, indem er einen textuellen Zusammenhang zwischen "Meine Preise" und "Wittgensteins Neffe" herstellt.

Ein anderes, sachbezogenes Beispiel für diese Stilisierung hat Reuter genauer untersucht: Bei ihm kann man nachlesen, wie Bernhard in der Erzählung „Die Ehrengabe des Kulturkreises des Bundesverbandes der deutschen Industrie“ die ,Tatsachen verfälscht':

Vor drei Tagen noch im Krankenbett, jetzt schon auf der Reise nach Regensburg, wo die Gotik auf dich wartet, dachte ich. Die Donau wurde immer noch schmäler, die Landschaft wurde immer noch lieblicher, schließlich, wo sie aufeinmal wieder öd geworden war und grau und fade, war Regensburg. Ich stieg aus und ging sofort in das Hotel Thurn und Taxis. Es war wirklich ein Hotel erster Klasse für eine Stadt wie Regensburg. Mir gefiel es und tatsächlich hatte ich mich in dem Hotel sofort wohl gefühlt, war ich doch von Anfang an nicht allein gewesen, sondern in Gesellschaft der Elisabeth Borchers, die ich schon einmal in Luxemburg getroffen hatte, auf einem der vielen sogenannten Dichtertreffen, auf die ich um die zwanzig herum mit meinen Gedichten gereist bin. (Bernhard 2009: 27 f.)

Reuter zeigt, dass man unter diesem Dichtertreffen die Mondorfer Dichtertage von 1968 versteht, denn Borchers nahm nur daran teil, aber die erzählte Preisverehrung fand ein Jahr früher, 1967 statt. Bernhard war übrigens zur Zeit der Dichtertage 37 Jahre alt und nicht „um die Zwanzig herum“, wie er es behauptet. Im Weiteren ist noch zu bemerken, dass er als Lyriker das letzte Mal im Jahre 1962 auftrat, denn danach war er als Schriftsteller in Mondorf präsent (vgl. Reuter 2013: 422).

Man darf die suggestive Wirkung der starken Bezogenheit auf die Wirklichkeit, die für Bernhard so charakteristisch ist, auch in "Meine Preise" nicht unterschätzen, und auch nicht vergessen, dass der Ich-Erzähler eine Maske, eine Rolle ist. Der Grundton des frühen Bernhards ist in diesem Sinne noch eindeutiger. Der Wechsel vom lyrischen ,Ich'zum erzählten ,Er' weist auf eine Verschiebung der Erzählperspektive hin, wobei man das Gefühl hat, dieselbe Betroffenheit des Erzählers zu lesen, nun aber aus einer bestimmten Distanz. Eine Distanz, die mit der erzählerischen Position der Ich-Perspektive in den autobiographischen Schriften sowie in „Wittgensteins Neffe" und in „Meine Preise“ in den Hintergrund gedrängt wird. Diese Perspektivierung zerstreut den Verdacht auf ein Rollenspiel. Bernhards Ich-Rolle hat eine fassbare Präsenz: Die Rhetorik der Kurzgeschichten in "Meine Preise“ baut sich stark auf diese 
Maske auf. Bernhard ist mit dem skandalösen Bild zufrieden, das im öffentlichen Leben über seine Person entstanden ist ${ }^{9}$ und verknüpft es geschickt mit seiner Ich-Rolle in "Meine Preise", indem er mit Erwartungen spielt, die durch das etablierte Bild über inn motiviert sind. Man bekommt eine Lektüre, wo verkehrte Umstände zu finden sind: In diesen Geschichten ist Bernhard derjenige, der die Etikette wahrnimmt, und die Politiker sind es, die gegen diese verstoßen. „Auch hier besteht Bernhards Strategie mithin darin, die Ereignisse so zu perspektivieren, als habe er selbst sich vollständig formatkonform verhalten und nur die Kulturpolitiker gegen Regeln verstoßen“ (Pethes 2016: 166). Die Selbstironie und die Situationskomik sind dieser Rhetorik ebenfalls untergeordnet. In „Grillparzerpreis“ gibt es ständig eingefügte Andeutungen darauf, dass der Erzähler mit den Erwartungen und Konventionen völlig im Klaren ist: „Zur Verleihung des Grillparzerpreises der Akademie der Wissenschaften in Wien [...] hatte [ich] plötzlich [...] eingesehen, dass ich zu dieser zweifellos außerordentlichen Zeremonie nicht in Hose und Pullover erscheinen könne [...] und so hatte ich [...] den Entschluß gefaßt [...] mich entsprechend feierlich einzukleiden“ (Bernhard 2009: 7). In der Geschichte bleibt dann diese Berücksichtigung der Formalitäten einseitig:

Die Freunde ließen uns in Ruhe und wir schauten uns in der Halle nach einer Persönlichkeit um, die uns empfangen würde. Ich ging ein paarmal mit meiner Tante in der Eingangshalle der Akademie hin und her, aber kein Mensch nahm von uns auch nur die geringste Notiz.

Diese Selbstinszenierung und eigenartige Perspektivierung sind eine Quelle des Humors, eine Rechtfertigung des Erzählers und ein rhetorischer Tragpfeiler, worauf Bernhard seine Geschichten in "Meine Preise" aufbaut.

Es ist vielsagend, dass die Werkausgabe (22 Bände) eine breite Palette von Bernhards Texten aufzeichnet: Sie beinhaltet Gedichte und Zeitungsartikel, juristische Berichte, Kurz- und Langprosa, Dramen und autobiographische Schriften. Es ist nicht herkömmlich, dass sogar Preisreden, autobiographische Erinnerungen und Feuilletonbeiträge in das Gesamtwerk eines Autors aufgenommen werden, aber es beweist, dass "Meine Preise " "nicht nur Satellit oder Beiwerk des literarischen Werkes" (Bombitz 2019: 38), sondern ein kontinuierlicher, organischer Teil dessen ist. Es bleiben nun die Fragen offen, wo die Grenze zwischen Literatur und ,Geschichte' liegt, und ob alles ,Mitgeteilte' von Thomas Bernhard als Literatur betrachtet werden kann.

9 „Bernhard hat es aber offensichtlich, wie nicht zuletzt die Rezensionen zu ,Meine Preise' belegen, durch ein gekonntes Spiel auf der Medienklaviatur geschafft, sich als Opfer des korrupten Staates Österreich und seiner ignoranten Öffentlichkeit zu positionieren und damit (werbetechnisch gesprochen) seinen Markenkern gefunden" (Huber 2016: 22). 


\section{Literatur}

Bernhard, Thomas (1978): Was Österreich nicht lesen soll. Die Kleinbürger auf der Heuchelleiter. 17. Februar 1978. https://www.zeit.de/1978/08/wasoesterreich-nicht-lesen-soll (abgerufen am 06.09.2020).

Bernhard, Thomas (2009): Meine Preise. Frankfurt am Main: Suhrkamp.

Bombitz, Attila (2019): Ergänzungen, Errettungen: Über eine ungarische Thomas-Bernhard-Ausgabe. In: Adamik, Lajos/Kovács, Edit (Hg.): Fogosch oder Zander. Kulturelle und mediale Übertragungen der Texte von Thomas Bernhard. Wien: Praesens, S. 28-46.

Huber, Martin (2016): „, beinahe alles falsch“? Dichtung und Wahrheit in Thomas Bernhards „Meine Preise“. In: Text+Kritik. Zeitschrift für Literatur 43, S. 12-35.

Klug, Christian (1991): Thomas Bernhards Theaterstücke. Tübingen: Metzler. https://doi.org/10.1007/978-3-476-03378-9

Menasse, Robert (2005): Das war Österreich. Gesammelte Essays zum Land ohne Eigenschaften. Hg. von Eva Schörkhuber. Frankfurt am Main: Suhrkamp.

Mittermayer, Manfred (2006): Thomas Bernhard. Frankfurt am Main: Suhrkamp.

Pethes, Nicolas (2016): "glauben Sie mir". Die Ausweitung der literarischen Kampfzone in Thomas Bernhards Interviews, Briefen, Preisreden und Feuilletonbeiträgen. In: Text+Kritik. Zeitschrift für Literatur 43, S. 155-172.

Reuter, Tim (2013): „Vaterland, Unsinn“. Thomas Bernhards (ent-)nationalisierte Genieästhetik zwischen Österreich-Gebundenheit und ÖsterreichEntbundenheit. Würzburg: Königshausen \& Neumann.

Sarankó, Márta (2009): Thomas Bernhard díjai [Thomas Bernhards Preise]. 12. Februar 2009, http://www.litera.hu/hirek/thomas-bernhard-dijai (abgerufen am 29.01.2020). 